\title{
DECISION MAKING WITH UNCERTAINTY
}

\author{
Qing Zhou ${ }^{\mathrm{a}}$, Wei Peng ${ }^{\mathrm{b}}$ \\ "the Software Institute, Zhongshan University, Guangzhou, Guangdong, 510275, \\ P.R. China \\ Email address: Inszq@zsulink.zsu.edu.cn \\ ${ }^{b}$ Sci. and Tech. Institute of GRC, 2 Gonghexi Rd., Guangzhou, Guangdong, 510060, \\ P.R.China \\ Email address: pengwei99@tom.com
}

Abstract: In this paper we discuss decision making based on incomplete knowledge. Asthe results of our decisions have always some uncertainties in these cases, we developeda method to measure the advantages brought by those possible solutions of our problem, which makes our decision making problem easier. Our proposal is entirely formulizedwithin the so called classical two valued logic, so it has a solid foundation. Basic notionsof various items are defined formally; formulas of supporting degree and safe supporting degree for decision making are discussed in details. Uncertainty of a proposition is clearly defined and the evaluation of such an uncertainty is clearly presented within our proposal without anything else. With the right evaluation of uncertain evidences, decision making with uncertain evidences is considered, which is also completely done within our proposal. Examples in the paper are comprehensively exhibited, which show that our proposal is reasonable and can be implemented by computers.

Key words: Reasoning under Uncertainty, Decision Support Systems

\section{INTRODUCTION}

In this paper we propose an auto-decision making system which is based on the knowledge we have and entirely formulized within the classical logic. Also the proposal in the paper can completely be implemented by computers.

The "decision making" problem can be described as following: We have a problem $p$, which could be our goal, or a difficulty we have to overcome. To reach our goal or overcome the difficulty, we have a few choices $c_{1} ; \ldots$; 
$c_{\mathrm{n}}$. which are supposed to be the possible solutions on the problem. The decision making problem is to find the best solution from the choice set $C=$ $\left\{c_{1} ; \ldots ; c_{\mathrm{n}}\right\}$.

In literature a number of research papers have been published in the area of decision making. Many of them use probability theory to work in this area, but probability is not always reliable in our practice. So other approaches are desirable.

From our view point, the knowledge on the problem $p$ is often more reliable than experiments or past experiences, so we attempt to use it in our proposal. To this end, let us take a look at what we should do when we have to make a decision.

Suppose we have a choice set $C=\left\{c_{1} ; \ldots ; c_{n}\right\}$ and we have to make a decision for the problem $p$. As the result of our decision is about what will happen in the future and all the knowledge we have is about what have already happened by now, there are always uncertainties on what will exactly happen. This makes decision making di \pm cult. To reach the best solution a common way often taken by experts is:

i) gather information on the problem as complete as possible;

ii) make "what ... if ..." analysis for every $c_{i} \in \mathrm{C}$ based on the knowledge gathered, i.e. use the knowledge we have in the specific area and the information gathered to see what advantages we can have from $c_{\mathrm{i}}$ for each $c_{i}$;

iii) compare the results of the above analysis for all $c_{i}$, and take the one as the decision which can bring us the most advantages than all others in $C$.

We will describe such a process formally and develop a method to select the best choice from the choice set C: For this purpose, a few notions are essential. First we have to have knowledge in the specific field in which the problem $\mathrm{p}$ is involved. Then we need information on $\mathrm{p}$, which is called evidence in this paper. These knowledge and evidences can usually be formulized into a first order logical system. So in this paper we develop our proposal within a first order reasoning system and divide the axiom set of the first order reasoning system into two parts: the corpus of knowledge and the evidence set, for convenience.

\section{References}

[1].Doyle, J., Maintenance and Belief Revision, in Belief Revision, Ed. by P. G"ardenfors, Cambridge University Press, 1992.

[2].Kanal, L., Lemmer, J.F., Uncertainty in Artificial Intelligence, North-Holland, Amsterdam, New York, Oxford, Tokyo, 1986.

[3].Zhou, Q., The Concept of Uncertain Reasoning and its Supporting Degree, Intelligent Systems,Proceedings of the ISCA 9th International Conference, 2000.

[4].Russell, S, Norvig, P. Artificial Intelligence - A Modern Approach, Chapter 16 "Making Simple Decisions", Prentice Hall, 1995 